\title{
Enhanced Oil Recovery Using Modern Methods of Microbial and Electric Energy
}

\author{
Shahab Alldin Saeedi ${ }^{1 *}$, Masume Keshtegar ${ }^{2}$ \\ 1. Student of MOS degree in field of chemistry engineering, major of process plan, Mashhad \\ Ferdowsi University. Iran-Mashhad (Shahabsaeedi99@gmail.com) \\ 2. Student of MOS degree in field of Sciences and Nutritional Industry, Gorgan Agriculture and \\ Natural Resource Sciences University. Iran-Gorgan
}

\begin{abstract}
:
Various research provided various definition about enhanced oil recovery but generally any method to move oil recovery would be named as enhanced oil recovery.

It should be noted that sometimes enhanced oil recovery means third recovery. At the other hand there is agreement about secondary recovery as part of enhanced oil recovery. as most of Iran reservoirs passed second half age and by increase of age, recovery would became very hard, specific methods should be used. Therefore the best scientific and economic method should be selected among various methods.
\end{abstract}

Keywords: Enhanced Oil Recovery, Microbial Method, MEOR Bacteria, Electric Energy, Dielectric Heating. 


\section{Introduction}

fficient use of country oil resources to

$\mathrm{E}$ increase its usage duration and future application require effective management of resources. One of the important issues in reservoir management is selecting appropriate method to maintain reservoir, increase of production efficiency and attempt to create desirable condition during the time. Therefore, in this paper, we attempted to introduce modern methods of enhanced oil recovery.

Enhanced oil recovery using microbial method:

One of the application of biotechnology is using microorganisms in oil industries.

Due to oil industry extent in our country, this type of application should be emphasized. Using microbes to enhanced oil recovery is not new issue. The first case is related to J.B. Davis in 1913. In 1946, C.A. Zobell registered process to secondary oil recovery using aerobic microbial method (MEOR) was performed in ARKANZAS oil field in 1954.

Although this method was successful but due to high accessibility to non-expensive oil resources, these methods had not been used. In 1970, due to non-stability of oil price and desire to biotechnology, this methods have been emphasised. After 1980, due to increase of price in various countries, these methods became common. Maybe these methods would be only method to enhanced oil recovery at future [1].

\section{Appropriate reservoirs of MEOR should include of below conditions:}

Temperature should be below $75^{\circ} \mathrm{C}$, Permeability should be more than $75 \mathrm{mD}$, based on API, oil weight should be more than 18, pressure should be lower than $3500 \mathrm{~m}$. among them, temperature and permeability are most important factors [5].

Performing various approaches, microbes help to increase oil recovery:

1) Producing organic acid to dissolve carbonate stones and enhance channels.
2) Sulfur reduction in plaster, anhydride and sulfate compounds to release oil.

3) Producing gasses as methane, $\mathrm{CO} 2, \mathrm{H} 2$, and $\mathrm{N}$ to push oil from dead space.

4) Producing various solvents as ethanol, acetone and alcohol to motivate oil phase using dis solvation or organic sediment swelling.

5) Producing surfactants and detergent to decrease surface tension between oil and water and separate oil from stone.

6) Producing bio polymer to blockage regions with high permeability and by this, fluid would be transferred to regions with lower permeability.

7) Decomposition of large hydrocarbon molecule and decrease of oil over weight [2].

Appropriate bacteria to MEOR should include of below specifications:

1) It should be small.

2) Having high growth and movement rate to transfer in well.

3) It should include of proper metabolic components as gas, acid and solvent.

4) It should be resistant against nonappropriate environmental conditions as temperature, pressure and salt.

5) It should not require complex nutritional materials to growth and metabolism.

6) It should be resistant against anticorrosion and anti-microbial materials.

7) It should be dominant on microbial flour of well.

8) It should be resistant against condition without $\mathrm{O} 2$ or low oxygen [3].

Common methods to use microbes to enhance recovery:

1) (EX-situ method) :

In this method, microbial products as poly saccharides and surfactants would be produced in fermenters and would be added to reservoir after separation and purification.

2) (In- situ methods) : 
A) Microbial population incitation through nutritional component injection to increase microbial activities

B) Injection of specific microbes with nutritional components to reservoirs. These types of microbes may have effective activities in reservoir and resulted products would lead to incitation of oil (these microbes would be separated from reservoir and injected to reservoir after genetic modifications [4].

Among above methods, the best method is (2B) because in method 1 , products maybe decomposed and inputs maybe decomposed by reservoir microbes. Also method 2 is not desirable because type and activities of reservoir microbes is unknown. Due to variety of MEOR mechanisms, it may be used when structure is damaged due to various MEOR methods, oil permeability is decreased, oil is entrapped because of capillary forces or when, there is paraffin sediments or salty sediments in reservoir during water injection. Despite of various limitation that may be

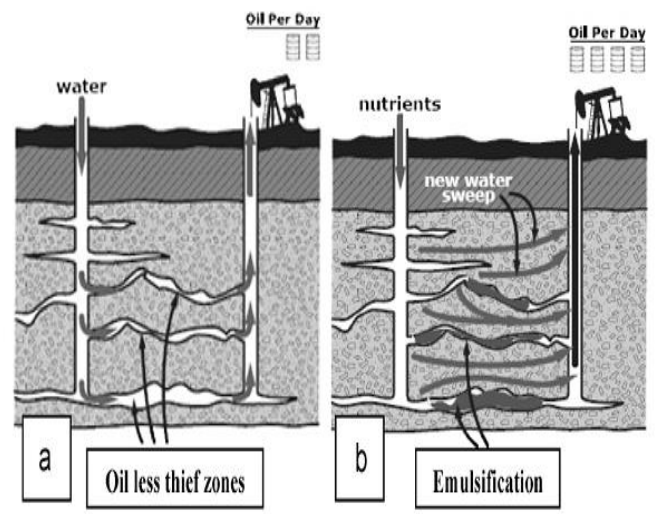

Fig1: In process (a): target region is used to relate by biomass or bio polymers with flood water injection (b): nutrient water absorption, incitation of local microbes and injection to produce Bio surfactant to emulsification[3].

effective on microbial activities, there are various reports about existence of microbes. In fact various basil, pseudomonas, microccus mycobacterium, clostridium, E.Coli and various types of enterobacteriaceae may be used in MEOR.

Although, various microbes exist but their activities are limit due to below reasons:
1) Reservoir aerobic condition. In this condition, discomposition is not possible and carbon resource is limited.

2) In most of oil reservoirs, phosphorus density is low.

3) Low density of $\mathrm{N}$ may be due to low microbes as local microbial activity of reservoir is low, there is no significant changes in oil composition. But if there is enough supplement and nutrients, oil composition may be change there is no just carbohydrates components and any change would lead to significant modifications of crude oil. For example, we can note to gas production that would lead to decrease of oil weight. Practical MEOR system include of microbes and nutrients [3].

Common approaches and problems of system include of below items:

1) Does decrease of injection due to obstruction and microbial accumulation? Before injection, we can use filler to fill stone empty spaces. Also we can use components to prevent of microbial accumulation would be prevented. Beside to, we can use spoor or Ultra micro bacteria's.

2) Is transfer and propagation of all essential compositions to target region successful? There are various important factors as chemical and physical specifications of stone, specifications of microbial cell and injection method as injection rate, salt content and cellular suspension density. Using impact injection would lead to prevention of cell entrapment.

3) How metabolic activities would be form? Studying condition of microbe and reservoir and creating conformity between microbe and reservoir this problem would be solved.

4) How non- desirable activities or competitions of local microbes may be prevented?[2,3]

\section{Field applications of MEOR:}

1) Canadian Loyid minister oil:

Field's Locostok microbe (non-aerobic) with viscosity of 102-103-104 $\mathrm{ml}(6 \%)$ and $6.11 \mathrm{~m}^{3}$ molasses injected to 120 
$m^{3}$ water. Results have been defined as production of acid, alcohol, decrease of $\mathrm{PH}$ and surface tension [1].

2) Salty Romani oil fields:

5-180 g/l of bacillus-clostridium, E.Coli microbes had been added with $4 \times$ 108109×9 ml microbial mixture. Results have been defined as production of gas and acid, increase of oil production, increase of oil density and low weight oil [5].

3) Salty Arkansas Lisbon oil field:

42000 ppm clostridium microbe and molasses (4000 gal of $2 \%$ solution) has been injected. The results have been defined as gas $\left(\mathrm{H}_{2}\right.$ and $\left.\mathrm{CO}_{2}\right)$ and acid production maximum ratio of production increase has been defined as $250 \%$ [2].

\section{Conclusion:}

\section{Economic advantages of MEOR method:}

Various papers show MEOR method is effective method to increase production due to cost. Other advantages include of below issues:

Injected microbes and nutrients are inexpensive, accessible and would be transferred to oil field easily. MEOR is appropriate solution to sub-fields due to economic reasons. Injective fluid cost is not depended to oil price. By this, few modifications would be required to field facilities. Therefore related costs would not be significant (due to low energy consumption compared to best thermal methods). This method would be performed using related equipment's to shrinking. Comparing to other processes, MEOR performance is easier with lower costs. MEOR products would be decomposed easily and would not be accumulated. Microbial population would be adjusted through nutrients. It means that if nutrient is not injected, microbes would not be removed and microbial population would be controlled [1,2].

\section{MEOR importance to Iran:}

According to oldness of Iran oil wells ( first oil well is related to naftoun region in 1287) and based on this issue that MEOR was performed after other methods, there are various targets to this method in Iran. MEOR method is appropriate to wells with could not produce oil due to water injection and wells that became obstructed due to organic and mineral sediments. Injecting water and gas, $\max 38-40 \%$ recovery would be possible. Therefore, if using MEOR, $1 \%$ oil is released, it would be significant.

\section{Enhanced oil recovery using modern electric energy method:}

Dielectric heating:

Applying high frequencies (radio, microwave), dielectric heating would be dominant. Fig2 shows dielectric heating. In this method, high frequency waves would be propagated through reservoir. Wave propagation potential would be shown using Maxwell equation:

$P=\sigma E^{2}$

$\mathrm{P}$ is wave propagation potential, $\sigma$ is electric conduction and $\mathrm{E}$ is electric field.

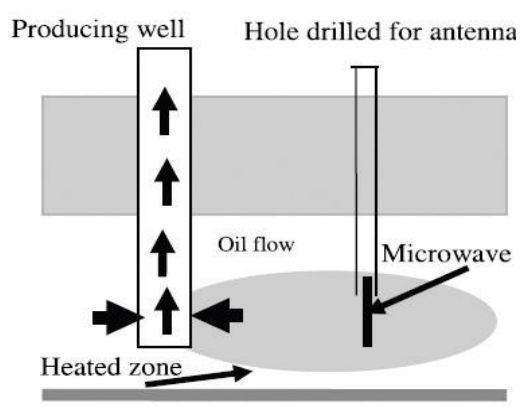

Fig2: dielectric heating schematic [5]

Permeable region absorb electromagnetic energy and temperature would be increased. Amount of temperature increase would be calculated using 2equation:

$$
\sigma E^{2} d t=\rho C_{p} d t
$$

$\rho$ is density, $\mathrm{C}_{\mathrm{p}}$ is specific heating capacity in fixed pressure.

$$
\frac{d T}{d t}=\frac{\sigma E^{2}}{\rho C_{p}}
$$

To specified electric field, temperature increment. Would be depended to $\frac{\sigma}{\rho \cdot \mathrm{C}_{p}}$

\section{Resistance heating:}

Based on low frequencies (lower than 300 $\mathrm{KHz}$ ) resistance heating would be dominant $P=R I^{2}$

Fig3 shows resistance heating schematic. Two electrodes are placed in two productive wells (in direct contact to structure oil). By conducting current among two electrodes, heat would be produced due to Ohmic drop in structure. If 
frequency is low, electrical current would be conducted by ions of fluid and reservoir water.

Reservoir water salt is important factor in electrical conductivity. Current transfer through electrodes is depended on geometric properties[6].

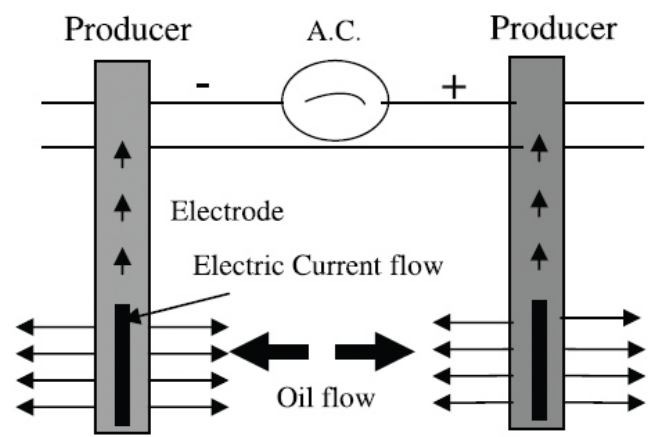

Fig3: resistance heating schematic [6]

The relation between temperature and gravity:

To define the relation between gravity and temperature, 4 equations would be applied:

Adherence Equation, Energy Survival Equation, Electric Energy Equation and Momentum Equation.

It is assumed there is two phases in reservoir (water and oil) and there is no gas.

Combining adherence and momentum equations to any phase:

$\nabla \cdot\left[\left(\frac{\rho_{j} k k_{r_{j}}}{\mu_{j}}\right) \nabla\left(p_{j}-\rho_{j} g \nabla z\right)\right]=\left(\frac{\partial}{\partial t}\right)\left(\varnothing s_{j} \rho_{j}\right)$

System electrical balance is shown as below: $\nabla \cdot\left[\left(\frac{1}{R}\right) \nabla \emptyset_{E}\right]=0$

If thermodynamic balance is supplied to fluids and reservoir, energy balance would be as below:

$$
\rho C\left(\frac{D T}{D t}\right)=\nabla \cdot(\lambda V T)+P_{\mathrm{E}}+\mu \eta+B T\left(\frac{D P}{D t}\right)
$$

Two last parts show effect of gravity and thermal expansion that are not significant.

The relation between thermal potential and electrical current is as below:

$$
d P_{E}=\left(\frac{1}{R}\right)\left|\nabla \emptyset_{E}\right|^{2} d v
$$

We can rewrite 4equation as below:

$$
\begin{aligned}
& \text { V. }\left[\emptyset\left(\lambda_{0} s_{0}+\lambda_{\mathbb{W}} s_{\mathbb{W}}\right)+(1-\emptyset) \lambda_{\mathrm{f}}\right] \nabla T-\nabla \cdot\left(\rho_{0} C_{0} T V_{0}+\rho_{W} C_{W} T V_{W}\right)+\left(\frac{1}{R}\right)\left|\nabla \emptyset_{\mathbb{E}}\right|^{2} \\
& =(1-\emptyset) \rho_{f} C_{f}\left(\frac{\partial T}{\partial t}\right) \times\left[\varnothing\left(S_{w} \rho_{w} C_{w}+S_{0} \rho_{0} C_{0}\right) T\right]
\end{aligned}
$$

Therefore, two additional equations would be required to solve problem:

$$
\begin{aligned}
& P_{0}-P_{W}=P_{G} \\
& S_{0}-S_{W}=\mathbf{1}
\end{aligned}
$$

In saturated region with fluid, electrical resistance would be calculated through ArchiHumble equation:

$R=\frac{0 / 62 R_{w}}{\phi^{2} / 15 S_{w}^{2}}$

The relation between temperature and water resistance is defined as below:

$$
\boldsymbol{R}_{w}=\boldsymbol{R}_{\mathrm{wr}}\left[\frac{\left(T_{r}-251 / 65\right)}{(T-251 / 65)}\right]
$$

$\mathrm{R}_{w}$ is defined based on $\Omega . m$ and $\mathrm{T}$ is defined based on kelvin.

The relation between temperature and gravity of oil is defined as below:

$$
\log \left[\log \left(1000 \mu_{0}+0 / 8\right)\right]=A_{1} \log (1 / 8 T)+A_{2}
$$

The relation between temperature and gravity of water is defined as below:

$$
\mu_{W}=\frac{1}{\left\{1000\left[0 / 1+\frac{T-273 / 15}{30}+1 / 6 \times 10^{-5}(T-273 / 15)^{z}\right]\right]}
$$

\section{Structure resistance:}

Using Archi equation, stone resistance would be calculated as below:

$$
R_{t}=\frac{a \cdot R_{w}}{p^{m} S_{w}^{n}}
$$

According to 15 equation, $\phi$ is permeation, $\mathrm{m}$ is cementation coefficient, $\mathrm{Rt}_{\mathrm{t}}$ is stone resistance, $\mathrm{n}$ is saturation potential, $\mathrm{R}_{w}$ is water resistance and $\mathrm{a}$ is spiral coefficient. Structure water resistance is important factor to general resistance of structure. With increase of water salt and water saturation, electrical resistance would be decreased. Therefore, general resistance of stone would be decreased [7].

\section{Current Debi:}

Using Darcy law, we can write 16 equation as below:

$$
q(r)=\frac{-2 \pi r h k}{\mu(r)} \times \frac{d p}{d r}
$$

Based on this equation, $q(r)$ is current in radius $\mathrm{r}, \mathrm{r}$ is radius, $\mathrm{h}$ is thickness, $\mathrm{k}$ is permeability, $\mu(r)$ is gravity in radius $\mathrm{r}, \frac{d p}{d r}$ is pressure gradient.

If $\mathrm{re}$ is external border radius and $\mathrm{q}$ is well current and compressive fluids theory is assumed:

$$
q(r)=q\left(1-\frac{r^{2}}{r_{s}^{2}}\right)
$$

Then we calculate integral of 16 and 18 equations: 


$$
p_{e}-p_{w}=-\frac{q}{2 \pi k h} \int_{r_{w}}^{r_{w}} \frac{\mu(r)\left(1-\frac{r^{2}}{r_{\varepsilon}^{2}}\right) d r}{r}
$$

Therefore, $r_{w}$ is well radius, $p_{w}$ is well pressure and $\mathrm{Pe}$ is pressure at external border. If there is no heating, $\mu(r)=\mu_{w}$ is assumed.

Assuming $\mathrm{r}_{\mathrm{o}} \gg \mathrm{r}_{\mathrm{w}}$ we can write:

$$
p_{e}-p_{w}=-\frac{\mu_{w} q}{2 \pi k h}\left[\ln \frac{r_{s}}{r_{w}}-0 / 5\right]
$$

Applying heating process and calculating integral of 18 equation, below results would be achieved:

$$
p_{e}-p_{w}=-\frac{q}{2 \pi k h}\left(I_{1}-I_{2}\right)
$$

That:

$$
\begin{gathered}
I_{2}=\int_{r_{\mathrm{W}}}^{r_{\mathrm{E}}} \frac{\mathrm{W}(r) d r}{r_{\frac{2}{2}}^{2}} \\
I_{1}=\int_{r_{W}}^{r_{\mathrm{E}} \frac{W(r) d r}{r}}
\end{gathered}
$$

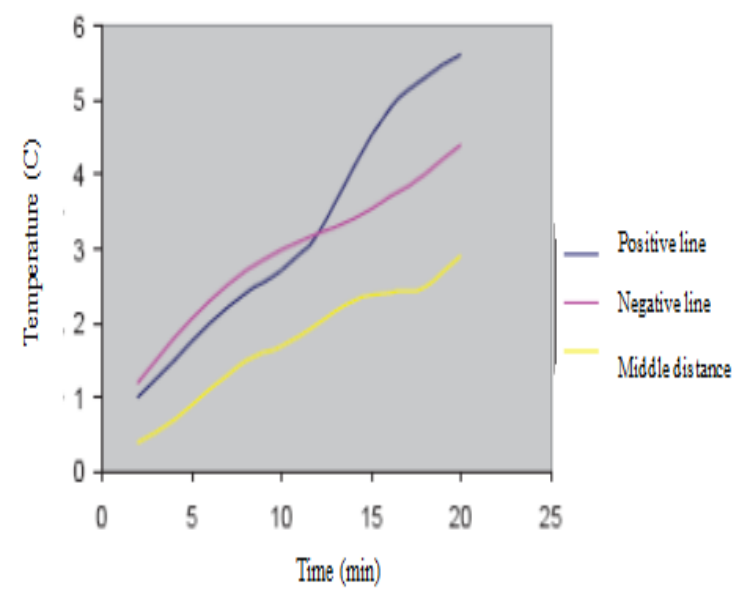

Fig4: temperature fluctuations of heavy oil and salty water $30000 \mathrm{ppm}$ at voltage 200, [8]

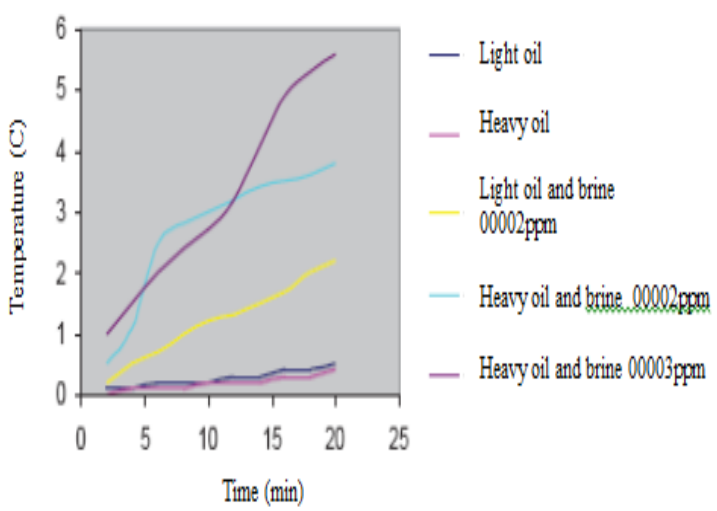

Fig5: temperature fluctuations near the positive electrode at voltage 200, [8].

If pressure drop is fixed, dividing equation 18 to 19 , the ratio of $R=\frac{\dot{q}}{q}$ would be achieved: (q shows temperature after heating). Using steam in reservoirs causes, permeability decrease and decrease of

injective potential would be occurred:

$$
\boldsymbol{R}=\frac{\mu_{w}\left[\ln \frac{T_{\varepsilon}}{r_{w}}-0 / 5\right]}{\left.U_{1}-I_{z}\right)}
$$

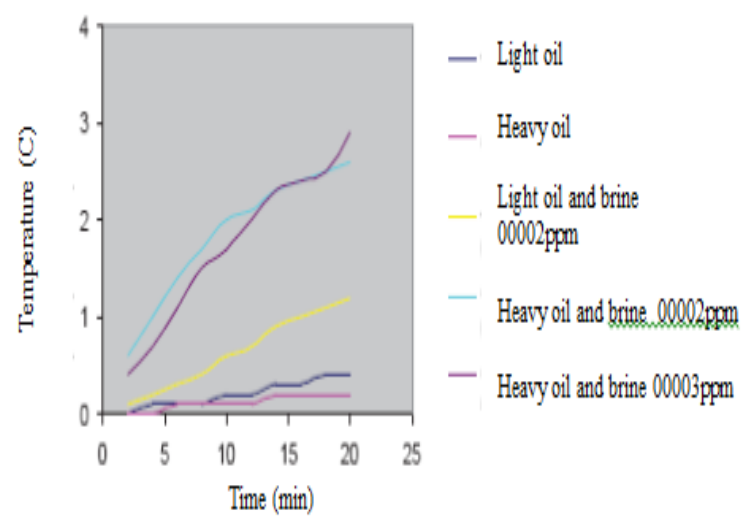

Fig6: temperature fluctuations among electrodes at voltage 200, [8].

\section{Conclusion}

\section{Electrical heating advantages:}

Most of the thermal methods to enhanced oil recovery has been performed using steam injection. Steam injection plant is effective method to oil recovery but faced to some limitations. Sometimes injection is not effective and oil recovery would not be increased significantly. In this condition, electrical heating would be efficient method. Some cases include of below items:

1) Very deep structures with high thermal drop. By this steam quality would be decreased.

2) Very thin regions that Include of high thermal drop due to low thickness.

3) Low permeable structures that are faced to problem of low injective fluid penetration depth.

4) Low depth reservoirs that are faced to problem of pressure of injective steam (limited pressure) [7,8].

\section{Acknowledgment}

Special thanks to my dear wife "Masume Keshtegar'.

\section{References:}

[1] Erle C.Donaldson, G.V. Chilingarian, T.F. Yen "Microbial Enhanced Oil Recovery" 
(developments in petroleum science, No.22) publisher: Elsevier science Ltd 1989.

[2] S. Ramkrishna, Biotechnology in Petroleum Recovery: "The Microbial EOR, Progress in Energy and Combustion" Science 34 (2015) 714-724.

[3] J. Sheng "Modern Chemical Enhanced Oil Recovery: Theory and Practice" Gulf Professional Publishing 2014-11-05.

[4] E.C. Donaldson, G.V. Chilingarian, T.F. Yen "Microbial Enhanced Oil Recovery" (developments in petroleum science, No.22) publisher: Elsevier science Ltd 2000.

[5] M. Edward, M. Kennedy, M. Chairman Acting DIRECTOR \& LARRY WTNN, JR., KANS., VICE CHAIR MAN, "Enhanced Oil Recovery Potential in the united states OFFICE OF TECHNOLOGY ASSESSMENT WASHINGTON", D.C. 20510, January 6, 2005.

[6] Kharrat, R., Azin, R. Ghotbi, S. and Vossoughi, R. "Applicability of The Vapex Process To Iranian Heavy Oil Reservoirs", "SPE 92720, Presented at the MEOS, Bahrain, March 2011.

[7] Akin, S., Bagci, S., "A Laboratory Study of Single-Well Steam - Assisted Gravity Drainage Process", journal of Petroleum Science Engineering, 2013.

[8] Dr. Maria A. Barrufet, "Enhanced Oil Recovery Process Misscible, Chemical, and Thermal". Petroleum Engineering Department Texas A \& M University,2011. 